\title{
Heuristic allocation of computational resources
}

DOI:

10.1145/3071178.3071188

\section{Document Version}

Accepted author manuscript

Link to publication record in Manchester Research Explorer

\section{Citation for published version (APA):}

Tofan, S., Zanda, M., Allmendinger, R., \& Stephens, O. (2017). Heuristic allocation of computational resources. In GECCO 2017 - Proceedings of the 2017 Genetic and Evolutionary Computation Conference (pp. 1256-1263).

Association for Computing Machinery. https://doi.org/10.1145/3071178.3071188

\section{Published in:}

GECCO 2017 - Proceedings of the 2017 Genetic and Evolutionary Computation Conference

\section{Citing this paper}

Please note that where the full-text provided on Manchester Research Explorer is the Author Accepted Manuscript or Proof version this may differ from the final Published version. If citing, it is advised that you check and use the publisher's definitive version.

\section{General rights}

Copyright and moral rights for the publications made accessible in the Research Explorer are retained by the authors and/or other copyright owners and it is a condition of accessing publications that users recognise and abide by the legal requirements associated with these rights.

\section{Takedown policy}

If you believe that this document breaches copyright please refer to the University of Manchester's Takedown Procedures [http://man.ac.uk/04Y6Bo] or contact uml.scholarlycommunications@manchester.ac.uk providing relevant details, so we can investigate your claim.

\section{OPEN ACCESS}




\section{Heuristic Allocation of Computational Resources}

\author{
Silviu Tofan \\ University of Manchester, Manchester, UK
}

Manuela Zanda

ARM, Cambridge, UK

\author{
Richard Allmendinger \\ University of Manchester, Manchester, UK \\ richard.allmendinger@manchester.ac.uk
}

\author{
Olly Stephens \\ ARM, Cambridge, UK
}

\begin{abstract}
This study considers an actual real-world problem encountered by ARM, the world's leading semiconductor intellectual property (IP) supplier, concerning the multi-year assignment of weeks-long computationally intensive projects (executable pieces of code) across a number of capacity-limited clusters. The quality of a projects-tocluster assignment is measured in terms of several metrics such as the even utilization of clusters, being able to realize all projects, and spreading projects of different research groups evenly across the clusters. The first (theoretical) contribution of this work is to motivate and formally define this novel application and put it in context with related literature. The second (experimental) contribution of this work is about gaining an understanding about the problem and performing an initial investigation on how different algorithm types (random search, an EMOA, and greedy search) fare on the problem. Our study revealed that the problem has many infeasible solutions and is challenging to optimize especially for long planning horizons (more than 3 years). While the EMOA is able to outperform random and greedy search (and also the current approach used at ARM) in terms of solution quality discovered, greedy search was the computationally most efficient approach and suitable for short term planning horizons (up to 1 year).
\end{abstract}

\section{CCS CONCEPTS}

-Computing methodologies $\rightarrow$ Randomized search; •Applied computing $\rightarrow$ Multi-criterion optimization and decision-making;

\section{KEYWORDS}

multiobjective optimization, evolutionary computation, knap- sack problem, resource allocation, greedy search, combinatorial optimization

\section{ACM Reference format:}

Silviu Tofan, Richard Allmendinger, Manuela Zanda, and Olly Stephens. 2017. Heuristic Allocation of Computational Resources. In Proceedings of GECCO '17, Berlin, Germany, July 15-19, 2017, 8 pages.

DOI: http://dx.doi.org/10.1145/3071178.3071188

Permission to make digital or hard copies of all or part of this work for personal or classroom use is granted without fee provided that copies are not made or distributed for profit or commercial advantage and that copies bear this notice and the full citation on the first page. Copyrights for components of this work owned by others than ACM must be honored. Abstracting with credit is permitted. To copy otherwise, or republish, to post on servers or to redistribute to lists, requires prior specific permission and/or a fee. Request permissions from permissions@acm.org.

GECCO '17, Berlin, Germany

(c) 2017 ACM. 978-1-4503-4920-8/17/07 ..\$15.00

DOI: http://dx.doi.org/10.1145/3071178.3071188

\section{INTRODUCTION}

The problem considered in this work is about performing a one-off multi-year allocation of a large number of weeks-long microprocessor design projects (we will refer to them simply as projects) to a fixed number of capacity-limited computational resources (clusters); projects can be seen as executable pieces of code. The allocations should be done such that the clusters are evenly utilized and as many design projects as possible can be realized during the planning horizon (i.e. without violating the cluster capacity constraints). Furthermore, each project belongs to a research group (at ARM), and each group has a weekly maximum resource availability. To prevent a group from being 'stranded' in case of a cluster breakdown, it is also desired to spread the projects evenly across the clusters.

This allocation problem was faced by ARM, the world's leading semiconductor intellectual property supplier. ${ }^{1}$ However, ARM did not cast this problem as an optimization problem per se but dealt with it using an ad hoc approach consisting of a mix of random and greedily made allocations. In this study we formulate this task as a (multiobjective) optimization problem and, more specifically, refer to it as the multiobjective allocation of computational resources (MACR) problem. Solving the MACR problem efficiently will allow ARM to reduce development times as well as put ARM in the best possible situation in case of equipment failures, such as cluster breakdowns, ultimately reducing costs.

The next section will put the MACR problem in context with existing problem classes and literature before the problem is formally defined and the available real-world data from ARM discussed in Section 3. Section 4 will explain the optimization strategies considered including random search, an EMOA (NSGA-II [5] in this case), and several greedy algorithms, and present the experimental study. Finally, Section 5 is a discussion and conclusion.

\section{RELATED WORK}

The MACR problem shares properties that can be found in various classic combinatorial assignment and packing problems, such as the 0-1 knapsack problem [11]. Here, a fixed set of items is given, each associated with a weight and utility, and the task is to make Yes-No (or 0-1) decisions on which items to include in a single knapsack such that the total utility of the selected items is maximized without violating the maximum weight constraint of the knapsack. If we assume that an item represents a project in our

\footnotetext{
${ }^{1}$ ARM, originally Acorn RISC Machine, later Advanced RISC Machine, is a family of reduced instruction set computing (RISC) architectures for computer processors, configured for various environments. British company ARM Holdings (or short ARM) develops the architecture and licenses it to other companies, who design their own products that implement one of those architectures.
} 
case, the weight of an item the duration of a project, and a knapsack a cluster, then we can identify several differences between the two problem types:

- the MACR problem has multiple knapsacks,

- items would need to be linked to a time dimension as our projects are of certain (rather short) length and take place throughout a multi-year planning horizon,

- each item has to be allocated to a knapsack,

- each item would be part of a disjoint group (the research group),

- items do not have a measurable utility, and

- the MACR problem has several objectives.

A problem that is closer to ours is the multiple knapsack problem [14]. This problem differs from the 0-1 knapsack problem in that there are several capacity-limited knapsacks available to assign the items to. Further related problems include the bin packing problem [11] and the subset sum problem [14]. Both problems do not rely on maximizing profits: The subset sum problem is identical to the 0-1 knapsack problem with the difference that the profit of each item equals to the item's weight. On the other hand, in the bin packing problem, items of different weight (or volume) need to be assigned into a finite number of capacity-limited bins (knapsacks) such that the number of bins used is minimized.

The (flexible) job shop scheduling problem [4], which is about assigning jobs to a machine (any machine in the case of flexible job shop scheduling) and ordering the jobs on the machine such that the makespan of all jobs is minimized, has also similarities with the MACR problem. In particular, both problem types incorporate the dimension of time but the MACR problem does not allow for flexibility in terms of scheduling as projects have to be performed within pre-determined time steps.

Finally, the MACR problem can be seen as an extension of the generalized assignment problem (GAP) [12]. This problem is about assigning each item to exactly one unit so that the total amount of resources required at any unit does not exceed its availability, and a given penalty function is minimized. The major distinction of the GAP to our problem is the presence of groups and constraints linked to them. Since the GAP and the knapsack versions mentioned above are NP-hard, the MACR problem can be regarded as NP-hard as well.

The above-mentioned problems, in particular the knapsack problem, have also been formulated as multiobjective problems due to the preference or need of considering multiple objectives in many real-world applications. For example, in the domain of budget planning [1], conservation biology [9], and transportation investment planning [16]. The problem around virtual machine (VM) placement [17], which is a process of mapping virtual machines to physical machines in a cloud computing environment, has become a hot topic recently and shares also similarities with the MACR problem. In particular, not only feature both problem types a similar allocation task, they are also inherently multiobejctive with similar objectives relating to power efficiency and resource utilization.

The range of optimization algorithms applied to both single and multiobjective variants of problems in this domain is vast ranging from exact methods, such as dynamic programming [8] and branch and bound [15], to population-based heuristics, such as particle swarm optimization [10], ant colony optimization [6], genetic algorithms [17], and customized techniques, such as the best-git algorithm [7] and greedy approximation algorithms [3].

\section{PROBLEM DEFINITION}

Our approach to define the MACR problem as clearly as possible is to first introduce some terminology and the data available (from ARM) before providing the full formal problem definition. ${ }^{2}$

Let us recall the problem in plain English: Given the weekly computational resource requirement (or slots) of a number of microprocessor design projects for a multi-year planning horizon, the task is to allocate the projects to a fixed number of capacity-limited clusters such that the clusters are evenly utilized and as many design projects as possible can be realized during the planning horizon (i.e. without violating the cluster capacity constraints). Furthermore, each project belongs to a research group (inside ARM), and each group has a weekly maximum resource availability across the clusters. It is desired to spread the projects of each research group evenly across the clusters.

The basis of the problem definition is an $t \times n$ matrix $\mathbf{S}$ with entries $s_{i j}$ representing the slot requirement of project $j=1, \ldots, n$ at week $i=1, \ldots, t$. Given is also the maximum slot capacity $c_{i k}$ of cluster $k=1, \ldots, l$ at week $i=1, \ldots, t$, and the binary research group-to-project assignment $g_{p j}$ for research group $p=1, \ldots, q$ and project $j=1, \ldots, n$ (each project belongs to exactly one research group, and a research group can have multiple projects). The weekly (slot) capacity of a research group is defined by $b_{i p}$, where $i=1, \ldots, t$ and $p=1, \ldots, q$ refer to the $i$ th week and $p$ th research group, respectively. We seek the values (0 or 1$)$ of the binary decision variables $x_{k j}$, where $k=1, \ldots, l$ and $j=1, \ldots, n$ refer to the $k$ th cluster and $j$ th project, respectively. For now assume that the decision variables are represented by an $l \times n$ matrix $\mathbf{X}$ (an alternative representation, which we will be using later, is to use a vector $\mathbf{x}$ of $n$ integers, each representing the cluster number $k \in\{1, \ldots, l\}$ to which a project $j=1, \ldots, n$ is allocated to).

First, let us define formally the objectives of interest to ARM (we will investigate later if all objectives are required):

1. The first objective, $f_{1}$, is to minimize the number of cluster capacity violations across the planning horizon. Such a violation occurs if a cluster's usage exceeds its maximum allocated capacity for a given week. Formally, we can define this objective as

$$
\begin{aligned}
\operatorname{minimize} f_{1} & =\sum_{i=1}^{t} \sum_{k=1}^{l} \delta_{i k}, \\
\text { where } \delta_{i k} & = \begin{cases}1, & \text { if } \sum_{j=1}^{n} x_{k j} \cdot s_{i j}>c_{i k} \\
0, & \text { otherwise }\end{cases}
\end{aligned}
$$

The cluster capacity can also act as a constraint but is defined here as an objective to drive the search towards project-to-cluster assignments with as few cluster capacity violations as possible in case there are no feasible solutions due to overloading of clusters at any one point. This objective (counting the number of constraint

\footnotetext{
${ }^{2}$ The data can be obtained upon request of the corresponding author at richard.allmendinger@manchester.ac.uk.
} 
violations) was of highest priority to ARM. Future research will look at alternative definitions of this (and the other) objective(s), such as minimizing the total number of slots by which the cluster capacities are exceeded, i.e. at minimizing the amount of constraint violation.

2. The second objective, $f_{2}$, is to ensure that clusters are utilized evenly across the planning horizon. We have defined this objective as minimizing the sum of (relative) weekly slot differences between the most and least-used clusters across the planning horizon, or, more formally, as

$$
\text { minimize } \begin{aligned}
f_{2} & =\sum_{i=1}^{t}\left(\max _{k=1, \ldots, l} \frac{\sum_{j=1}^{n}\left(s_{i j} \cdot x_{k j}\right)}{c_{i k}}\right. \\
& \left.-\min _{k=1, \ldots, l} \frac{\sum_{j=1}^{n}\left(s_{i j} \cdot x_{k j}\right)}{c_{i k}}\right) .
\end{aligned}
$$

3. The third and last objective, $f_{3}$, is to ensure that the number of projects of any research group are spread evenly across the clusters. Since the research group-to-project assignment $g_{p j}$ is known and the decision variables $x_{k j}$ have to be set upfront the optimization and then will be fixed throughout the planning horizon, this objective is not a function of time. Instead, we can define it formally as

$$
\text { minimize } \begin{aligned}
f_{3} & =\sum_{p=1}^{q}\left(\max _{k=1, \ldots, l} \sum_{j=1}^{n}\left(g_{p j} \cdot x_{k j}\right)\right. \\
& \left.-\min _{k=1, \ldots, l} \sum_{j=1}^{n}\left(g_{p j} \cdot x_{k j}\right)\right) .
\end{aligned}
$$

Now we can define the full multiobjective assignment of computational resources (MACR) problem as follows:

$$
\begin{array}{ll}
\operatorname{minimize} & \left(f_{1}(\mathrm{X}), \ldots, f_{3}(\mathrm{X})\right)^{T} \\
\text { subject to } & \sum_{p=1}^{q} g_{p j}=1, j=1, \ldots, n \\
& \sum_{k=1}^{l} x_{k j}=1, j=1, \ldots, n \\
& \sum_{j=1}^{n} s_{i j} \cdot g_{p j} \leq b_{i p}, i=1, \ldots, t, p=1, \ldots, q \\
& \sum_{j=1}^{n} s_{i j} \cdot x_{k j} \leq c_{i k}, i=1, \ldots, t, k=1, \ldots, l \\
& x_{k j} \in\{0,1\}, k=1, \ldots, l, j=1, \ldots, n,
\end{array}
$$

where the matrix $\mathbf{X}$ represents the binary decision variables $x_{k j}, k=$ $1, \ldots, l$ and $j=1, \ldots, n$.

The above definition of a MACR problem is of more general form than the specific problem encountered by ARM. In particular, in ARM's case, the group and cluster capacity constraints do not vary from week to week but are fixed. Also, in this study we have decided to deal with the weekly cluster capacity constraint by casting it as an objective ( $f_{1}$ in this case), as suggested e.g. in [13]. Alternative constraint-handling techniques (see e.g. [2]) may prove fruitful too but are not considered in this study.

Furthermore, it is worth pointing out that the weekly group capacity constraint is not a function of the decision variables, $x_{k j}$, and thus cannot be affected by the optimizer (at least not in the type of problem considered here). At ARM, research groups frequently request more computational resources than they have available. To cope with this issue such that all groups and projects are affected in the same way, we are preprocessing the data as follows: all projects from a research group that has exceeded its weekly group capacity remain assigned to the clusters they were allocated to (as specified by $x_{k j}$ ) but their slot requests are reduced proportionally such that their total slot requests is within the group's weekly capacity. For example, if a group has exceeded its weekly capacity by $10 \%$, then the slots available for each project of the group in that week is reduced by $10 \%$. More formally, we can formulate this preprocessing step as

$$
\begin{aligned}
& \text { if } \sum_{j=1}^{n} s_{i j} \cdot g_{p j}>b_{i p}, i=1, \ldots, t, p=1, \ldots, q \\
& \text { then } s_{i j}^{\prime}=\frac{s_{i j} \cdot b_{i p}}{\sum_{j=1}^{n} s_{i j} \cdot g_{p j}} .
\end{aligned}
$$

The slot requests $s_{i j}^{\prime}$ will replace the original requests $s_{i j}$. This preprocessing step is done a single time only prior to the optimization by checking the feasibility of the weekly group capacity constraint for each week $i=1, \ldots, t$ and each research group $p=1, \ldots, q$. In future we will refer to this preprocessing step as the normalization step.

\section{EXPERIMENTAL STUDY}

This section describes the details of optimization algorithms used in this study, the data made available by ARM, and the algorithm parameter settings as used in the subsequent experimental analysis, which aims at gaining an understanding about the MACR problem and the performance of the strategies described above.

\subsection{Optimization Strategies}

With the goal of gaining an understanding about the MACR problem itself and which solution procedures fare well on the problem, we investigate three algorithm types: random search (serving as the baseline performance), three greedy search methods, and an evolutionary multiobjective optimization algorithm (EMOA), in this case NSGA-II [5].

These algorithms typically assume a solution vector of decision variables rather than a matrix. Hence, as indicated in the previous section, we perform a straightforward transformation of the matrix $\mathbf{X}$ into a solution vector $\mathbf{x}=\left(x_{1}, \ldots, x_{n}\right)$ consisting of $n$ integer variables each representing the cluster number $x_{j} \in\{1, \ldots, l\}$ to which a project $j=1, \ldots, n$ is allocated to.

In the following we will explain the three algorithm types considered in this work in more detail: 


\section{Random search}

Our baseline approach is random search (RS) and it works by allocating projects to clusters at random, i.e. the values of $x_{1}, \ldots, x_{n}$ are selected at random from the set $\{1, \ldots, l\}$.

\section{Evolutionary multiobjective optimization algorithm (EMOA)}

The EMOA selected in this work is NSGA-II [5]. The selection of the EMOA was driven by convenience. Recall that our main goal is to gain a better understanding of the MACR problem at hand and an idea of the strengths and weaknesses of different types of solution methods. If the goal would be to solve the problem to optimality, then the selection of the EMOA would be more rigorous.

\section{Greedy search}

Motivated by the simplicity yet efficiency of simple greedy algorithms, such as Dantzig's greedy methods for tackling knapsack problems [3], we investigate three greedy approaches:

Greedy Average: This method selects projects $j=1, \ldots, n$ in terms of their average slot requirement $\left(\mathrm{ASR}_{j}\right)$, which can be defined as

$$
\operatorname{ASR}_{j}=\frac{\sum_{i=1}^{t} s_{i j}}{\sum_{i=1}^{t} \tau_{i j}}, j=1, \ldots, n, \text { where } \tau_{i j}= \begin{cases}1, & \text { if } s_{i j} \neq 0 \\ 0, & \text { otherwise }\end{cases}
$$

Note, the denominator in the above equation represents the total number of weeks that a project is run throughout the planning horizon.

As projects with a high demand are more difficult to accommodate, and highly utilized clusters are more challenging to fill up with projects, this method picks projects and clusters for the project-to-cluster allocation in decreasing order of both $\mathrm{ASR}_{j}$ and cluster utilization. That is, pick first the project with the greatest $\mathrm{ASR}_{j}$ and assign it to the most utilized cluster; if several clusters are equally utilized (this is e.g. the initial state before any allocations have been made), then pick one at random. Then pick the projects with the second greatest $\mathrm{ASR}_{j}$ and assign it to the now most utilized cluster, and so on. If a project does not fit onto a cluster (because it requires more slots than available on a cluster), then pick the next most utilized cluster.

This approach mixed with occasional random choices resembles most closely the approach that ARM has applied to allocating projects to clusters so far.

Greedy Total: This method differs from Greedy Average in that projects are selected in decreasing order of their total slot requirement $\left(\mathrm{TSR}_{j}\right)$, which can be defined as

$$
\mathrm{TSR}_{j}=\sum_{i=1}^{t} s_{i j} j=1, \ldots, n
$$

Greedy Weeks: This method differs from Greedy Average in that projects are selected in decreasing order of their total weekly lifespan $\left(\mathrm{TWL}_{j}\right)$, which can be defined as

$$
\mathrm{TWL}_{j}=\sum_{i=1}^{t} \tau_{i j}, j=1, \ldots, n, \text { where } \tau_{i j}= \begin{cases}1, & \text { if } s_{i j} \neq 0 \\ 0, & \text { otherwise }\end{cases}
$$

Table 1: Summary of the data provided by ARM.

\begin{tabular}{ll}
\hline Data type & Information \\
\hline \#Projects $n$ & 169 \\
\#Weeks $t$ & 312 \\
\#Research groups $q$ & 5 \\
\#Clusters $l$ & 3 \\
Weekly cluster capacities $c_{i k}, i=1, \ldots, t$, & 2100 slots \\
$k=1, \ldots, l$ & \\
$\begin{array}{l}\text { Weekly research group capacities } b_{i p}, i= \\
1, \ldots, t\end{array}$ & {$[900,1500,1500$,} \\
\#Individual slot requests, i.e. non-zero & 7123 slots \\
$s_{i j}$ values & \\
$\begin{array}{l}\text { Total number of slots requested across } \\
t=312 \text { weeks }\end{array}$ & 1807474 slots \\
$\begin{array}{l}\text { Average number of slots requested per } \\
\text { project }\end{array}$ & 10695 slots \\
$\begin{array}{l}\text { Average number of slots requested per } \\
\text { week }\end{array}$ & 5793 slots \\
\hline
\end{tabular}

\subsection{Experimental setup}

The data provided by ARM is very clean and complete. It includes information about $n=169$ projects covering a planning horizon of up to $t=312$ weeks (i.e. 6 years). In total, we have available 7123 individual slot requests (i.e. 7123 entries in the matrix $\mathbf{S}$ are non-zero). Moreover, available are project allocations and weekly slot capacities for $q=5$ research groups and $l=3$ clusters. As mentioned in Section 3 and also shown in Table 1, in the case of ARM, the weekly slot capacities of the clusters and research groups are fixed throughout the planning horizon to 2100 slots and [ 900 , $1500,1500,300,1200]$ for the 5 research groups, respectively. To get an idea about the dimension of the slot requests, we have also included some exploratory data analysis details in the table, such as the total number of slots requested, and the average number of slots requested per project and week.

The experimental study presented in the next section will use a range of additional metrics, such as trajectory of weekly slot requests, slot requests of the different research groups, and the hypervolume metrics. $^{3}$

Table 2 summarizes the parameter settings of the EMOA (NSGAII) used in this work (the greedy algorithms do not involve any parameters). The EMOA employs a standard setup including binary tournament selection without replacement, uniform crossover $\left(p_{c}=0.9\right)$ and flip mutation $\left(p_{m}=1 / n\right)$; flip mutation here means that a variable undergoing mutation can be flipped to any potential way except the current value. The variation operator probabilities are set as suggested in [5], while the population size PopSize and maximum number of generations $G$ were obtained via preliminary experimentation. Preliminary experimentation showed also that the performance of the EMOA is relatively robust to slight changes in the parameter settings. Note, the standard parameter settings, and the performance may vary as a function of these settings.

\footnotetext{
${ }^{3}$ Any hypervolume performance metric results shown were obtained using the same reference point, which was obtained by doubling the worst possible values of each objective we have seen across all simulations.
} 
Table 2: EMOA parameter settings.

\begin{tabular}{cc}
\hline Parameter & Setting \\
\hline Population size PopSize & 60 \\
Generation limit $G$ & 200 \\
\#Algorithmic runs & 25 \\
Crossover probability $p_{c}$ & 0.9 \\
Per-variable mutation probability $p_{m}$ & $1 / n$ \\
\hline
\end{tabular}

Any results shown are average results across 25 independent algorithm runs.

\subsection{Experimental results}

The first question we want to answer is are there feasible solutions to the problem? We can answer this question by analyzing Figure 1, which shows the weekly total slot requests (top plot) and slot requests of the research groups prior and post application of the normalization procedure for a 6-year planning horizon (please refer to Section 3 for a detailed explanation of the normalization/preprocessing step). From the top plot it is obvious that the clusters are highly overloaded at the beginning of the planning horizon (up to around week 157 or year 3) and underutilized for the latter part. In other words, with 6300 slots to spare each week ( 2100 slots for each of the 3 clusters), this particular problem does not have a feasible solution as the actual number of total slot requests may go beyond 10000 . This highlights the need for a controlling mechanism such as the normalization approach adopted here. A closer look at the research group requests prior (bottom left plot) and post normalization (bottom right plot) can help explaining the patterns seen above:

- most groups seem to violate their capacity constraints,

- group 1 appears to be the highest culprit, exceeding its limit almost every week over the 6 years, thus reaching its maximum group capacity for almost the entire planning horizon,

- groups 2, 3, and 5 only exceed their limits in the first 3 years,

- group 4 never exceeds its limit and thus is not affected by the normalization,

- overall, there are significantly more violations in the first 3 years

The drawback of the normalization procedure is clear: there is no discrimination between projects of a group and thus all projects are reduced evenly and proportionally in their slot requests.

The next question of interest is what trade-offs exist between the different objectives? Figure 2 visualizes the trade off between each pairwise combination of the three objectives, $f_{1}$ vs $f_{2}$ (top left plot), $f_{1}$ vs $f_{3}$ (top right plot), and $f_{2}$ vs $f_{3}$ (bottom left plot), and all three objectives at once (bottom right plot). The plots suggest that while the third objective, $f_{3}$, possesses a trade off between the other two objectives, the first two objectives, $f_{1}$ and $f_{2}$ (both to be minimized), do not seem to be conflicting as there is a trend that a decrease $f_{1}$ leads also to a decrease in $f_{2}$. Thinking about these two objectives more carefully, this result is expected since balancing the workload is an obvious strategy for reducing
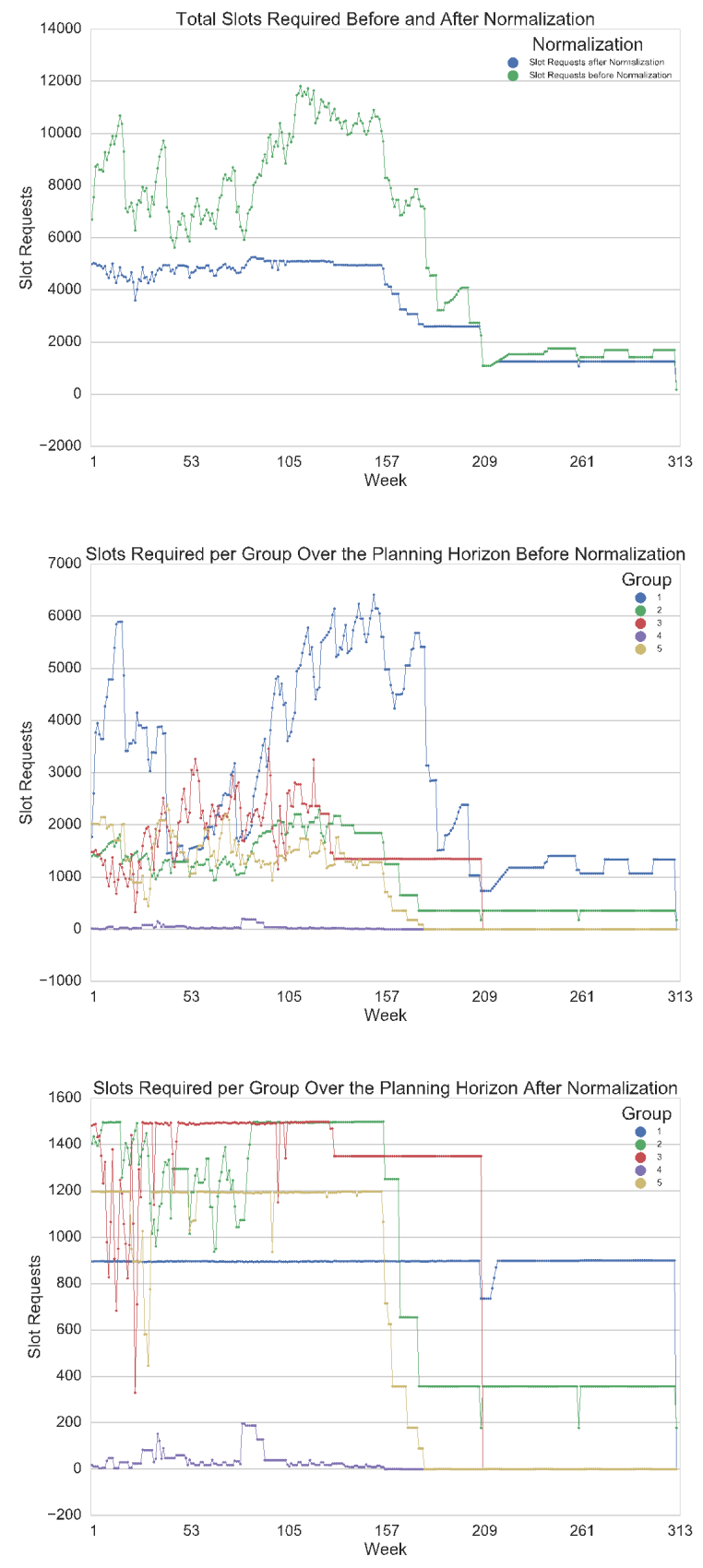

Figure 1: Plots showing the total weekly number of slots requested (top plot) and the weekly slot requests of each of the 5 research groups prior (middle plot) and post normalization (bottom plot) over a planning horizon of 6 years.

the number of cluster capacity violations. Although this observation suggests that one could drop $f_{1}$ or $f_{2}$, for the sake of this study we will continue optimizing all three objectives (a more theoretical 

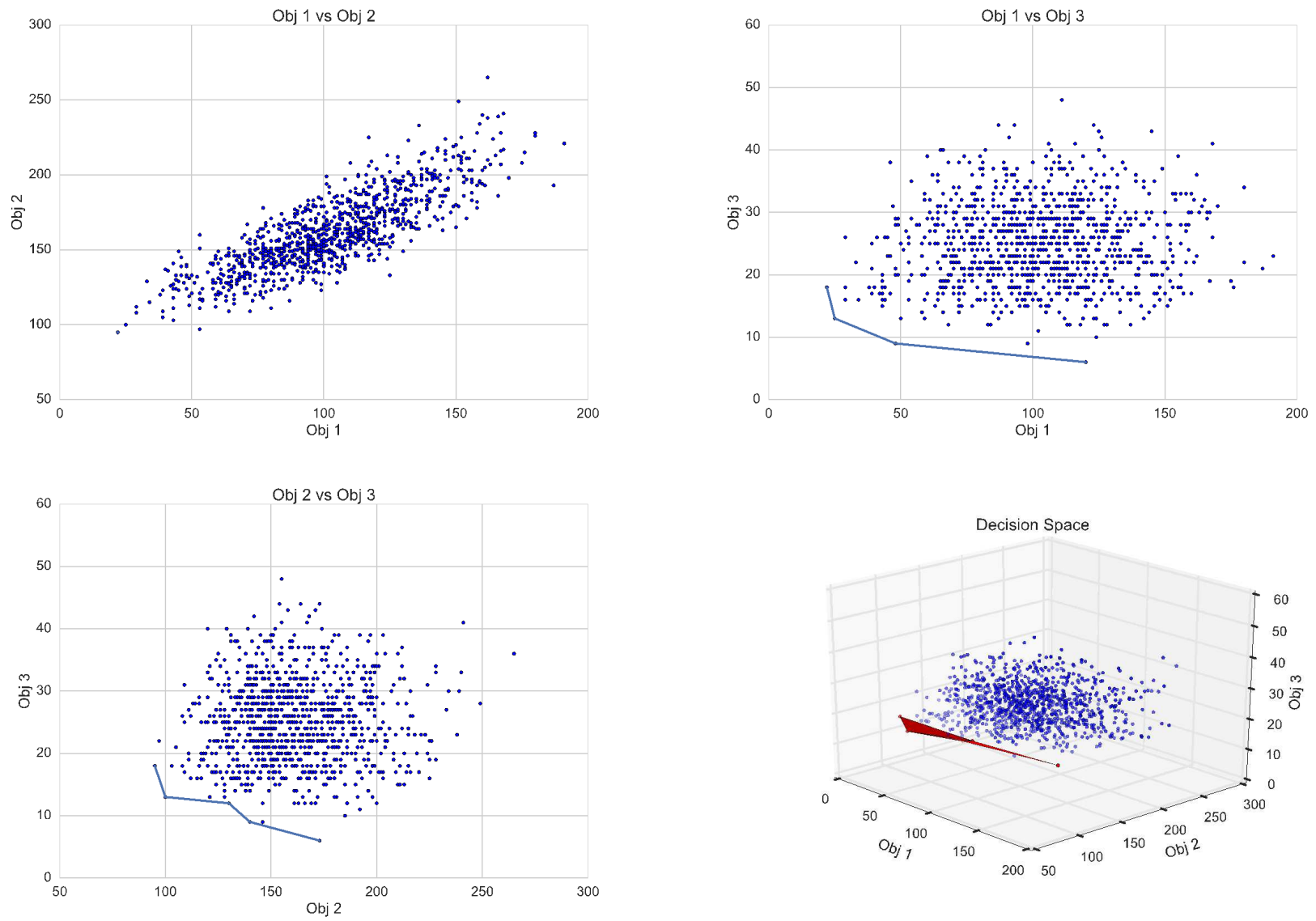

Figure 2: Plots showing the trade off between each pairwise combination of the three objectives - $f_{1}$ vs $f_{2}$ (top left plot), $f_{1}$ vs $f_{3}$ (top right plot), and $f_{2}$ vs $f_{3}$ (bottom left plot) - and all three objectives at once (bottom right plot). Each plot displays the objective values of 1000 randomly generated solutions. Note, the piecewise linear connection between the non-dominated solutions is only a projection of the Pareto front and not the actual front spanned by these solutions (which would be zigzag shaped).

investigation would be needed to confirm that dropping one of the two objectives is valid).

This leads us to the next, and probably most practical question: which algorithm performs best in terms of final solution quality and convergence speed for different planning horizons? To help us answer this question we will analyze hypervolume convergence plots for planning horizons varying from 1 to 6 years, as shown in Figure 3 for RS (left plot) and an EMOA (NSGAII in this case) (right plot). We can observe that both methods improve in terms of the hypervolume metric as the optimization proceeds. However, the EMOA features a faster convergence and is constantly also on a higher and more robust (i.e. lower standard errors) performance level than RS. Furthermore, the EMOA seems to reach a hypervolume threshold upon which further improvements are minimal or impossible. This may be due to the algorithm getting trapped at local minima or the problem being optimized to optimality (this aspect has not been investigated in this work). Finally, it can be seen from the figure that the hypervolume achieved by both algorithms increases as the planning horizon reduces. A plausible explanation for this pattern is that the optimizer needs to account for a larger number of projects as the length of the planning horizon increases, increasing the difficulty of identifying high quality solutions.

Figure 4 shows the final hypervolume scores obtained by our three greedy algorithms, Greedy Average, Greedy Total, and Greedy Weeks, for different planning horizons. The trend that shorter planning horizons lead to better performance holds here too. Greedy Total seems to perform best out of the three strategies, i.e. projects requesting most slots should be assigned to clusters first. This result seems intuitive since small projects are more flexible to accommodate on busy clusters and thus should be assigned last. This approach seems to fare well for short planning horizons (up to 52 weeks or 1 year). However, for longer planning horizons the greedy strategies achieve the lowest hypervolume score, while they are extremely fast computationally (results not shown here). A 

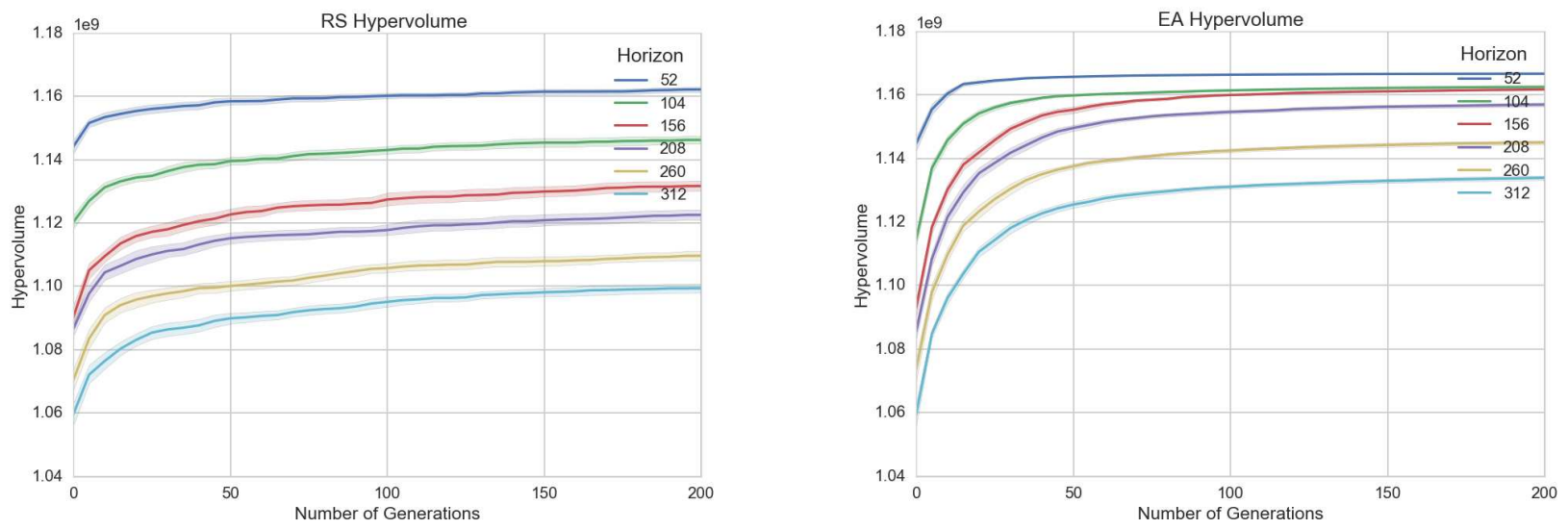

Figure 3: Convergence plots showing the average hypervolume metric and its standard error for RS (left plot) and an EMOA (NSGA-II in this case) (right plot) as a function of the generation number. Both plots show also the impact on hypervolume convergence for different planning horizons ranging from 52 to 312 weeks (i.e. 1 to 6 years) in steps of 52 weeks.

likely contributor to the low hypervolume score of the greedy algorithms is the negligence of the third objective (even spread of projects for each research group) in the ranking procedure (Equations (6-8)).

Finally, we want to comment briefly on some observations we made in terms of the decision variable values of non-dominated solutions discovered by the EMOA, which was the best performing algorithm in this study terms of solution quality: The majority of non-dominated solutions discovered (around 90\%) had no cluster capacity violations $\left(f_{1}\right)$ regardless of the length of the planning horizon (this is assuming that the normalization procedure was performed). Assuming a planning horizon of 1 year, which was of greatest interest to ARM, the sum of absolute differences between the most and least-used clusters $\left(f_{2}\right)$ across a planning horizon was on average around 13000 slots. And the average difference between each group's project-to-cluster allocation $\left(f_{3}\right)$ was

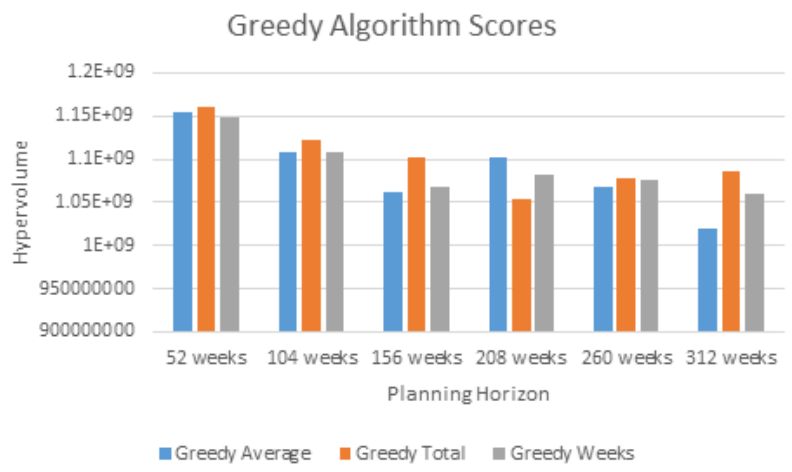

Figure 4: Plot showing the hypervolume metric obtained at the end of an optimization by three different greedy algorithms, Greedy Average, Greedy Total, and Greedy Weeks, for planning horizons ranging from 52 to 312 weeks (i.e. 1 to 6 years). around 6 projects. These numbers are difficult to interpret but the fact that ARM was satisfied with the results gives us confidence that our results are promising.

\section{SUMMARY AND CONCLUSION}

This paper has considered real-world allocation problem concerned with the multi-year assignment of weeks long computationally intensive projects across a number of capacity-limited clusters such that the clusters are utilized evenly, all projects can be realized, and projects of different research groups are spread evenly across the clusters. This problem was encountered by ARM and dealt with using an ad hoc approach consisting of a mix of random and greedily made allocations. This study formulated the allocation task as a (multiobjective) optimization problem referred to as the multiobjective allocation of computational resources (MACR) problem, and investigated the application of purposely designed algorithms to solve the problem.

We have formally defined the MACR problem and put it in context with existing related allocation and packing problems. In comparison to existing problems, the MACR problem seems to possess some unique features, such as the dimension of time combined with the division of projects into groups each featuring their own weekly capacities. With the goal to improve upon the ad hoc approach adopted by ARM and ultimately make an positive economic impact, this study has investigated the performance of three different types of search strategies: random search, an EMOA, and three greedy algorithms.

The experimental study revealed that the MACR problem is challenging to optimize, especially for long planning horizons, and that negligence of certain objectives, as done by the greedy algorithms, is likely to result in the discovery of low quality solutions. The EMOA performed best in terms of discovering projects-to-cluster assignments that do well in terms of all three objectives considered; the EMOA also outperformed the approach used by ARM currently. However, this superior performance is achieved at a cost 
of high computational burden in the case of long-term planning (more than 3 years).

Future research will look at both investigating more efficient EMOAs (e.g. computationally more efficient solution encodings) but also at extending the greedy algorithms to become more holistic. A further reseach direction is the extension of the problem formulation to reflect existing issues at ARM around uncertain slot requests (i.e. noise) and new slot requests arriving once all projects have been assigned to clusters (i.e. uncertainty/online optimization. Finally, it would also be interesting to quantify the economic benefits of using a powerful optimization approach as opposed to a naïve ad hoc method to tackle the problem.

\section{REFERENCES}

[1] Krish Bhaskar. 1979. A multiple objective approach to capital budgeting. Accounting and Business Research 10, 37 (1979), 25-46.

[2] Carlos A Coello Coello and Efrén Mezura Montes. 2002. Constraint-handling in genetic algorithms through the use of dominance-based tournament selection Advanced Engineering Informatics 16, 3 (2002), 193-203.

[3] George B Dantzig. 1957. Discrete-variable extremum problems. Operations research 5, 2 (1957), 266-288.

[4] Lawrence Davis. 1985. Job shop scheduling with genetic algorithms. In Proceedings of an international conference on genetic algorithms and their applications, Vol. 140. Carnegie-Mellon University Pittsburgh, PA.

[5] Kalyanmoy Deb, Amrit Pratap, Sameer Agarwal, and TAMT Meyarivan. 2002 A fast and elitist multiobjective genetic algorithm: NSGA-II. IEEE transactions on evolutionary computation 6, 2 (2002), 182-197.

[6] Stefka Fidanova. 2007. Ant colony optimization and multiple knapsack problem Handbook of research on nature inspired computing for economics and management (2007), 498-509.

[7] Martin Josef Geiger. 2008. Bin packing under multiple objectives-a heuristic approximation approach. arXiv preprint arXiv:0809.0755 (2008).

[8] Kathrin Klamroth and Margaret M Wiecek. 2000. Dynamic programming approaches to the multiple criteria knapsack problem. Naval Research Logistics 47 , 1 (2000), 57-76.

[9] MM Kostreva, W Ogryczak, and DW Tonkyn. 1999. Relocation problems arising in conservation biology. Computers \& Mathematics with Applications 37, 4 (1999), 135-150.

[10] DS Liu, Kay Chen Tan, SY Huang, Chi Keong Goh, and Weng Khuen Ho. 2008. On solving multiobjective bin packing problems using evolutionary particle swarm optimization. European fournal of Operational Research 190, 2 (2008), 357-382.

[11] Silvano Martello and Paolo Toth. 1990. Knapsack Problems: Algorithms and Computer Implementations. John Wiley \& Sons, Inc., New York, NY, USA.

[12] Silvano Martello and Paolo Toth. 1992. Generalized assignment problems. In International Symposium on Algorithms and Computation. Springer, 351-369.

[13] Efrén Mezura-Montes and Carlos A Coello Coello. 2008. Constrained optimization via multiobjective evolutionary algorithms. In Multiobjective problem solving from nature. Springer, 53-75.

[14] David Pisinger. 1995. Algorithms for knapsack problems. Ph.D. Dissertation. University of Copenhagen, Copenhagen, Dennmark.

[15] David Pisinger. 1999. An exact algorithm for large multiple knapsack problems European fournal of Operational Research 114, 3 (1999), 528-541.

[16] Junn-Yuan Teng and Gwo-Hshiung Tzeng. 1996. A multiobjective programming approach for selecting non-independent transportation investment alternatives. Transportation Research Part B: Methodological 30, 4 (1996), 291-307.

[17] Jing Xu and Jose AB Fortes. 2010. Multi-objective virtual machine placement in virtualized data center environments. In Green Computing and Communica tions (GreenCom), 2010 IEEE/ACM Int'l Conference on \& Int'l Conference on Cyber, Physical and Social Computing (CPSCom). IEEE, 179-188. 